\title{
Charge-Induced Ripplon Softening and Dimple Crystallization at the Interface of ${ }^{3} \mathrm{He}-{ }^{4} \mathrm{He}$ Mixtures
}

\author{
$\mathrm{M}_{\circ}$ Wanner and P. Leiderer \\ Fachbereich Physik der Technischen Universität Munchen, 8046 Garching, West Germany \\ (Received 4 December 1978) \\ The interface of phase-separated ${ }^{3} \mathrm{He}-{ }^{4} \mathrm{He}$ mixtures, when charged with negative ions, \\ shows a pronounced softening of its ripplon spectrum at wave vectors around the inverse \\ capillary length. As the electrical field perpendicular to the interface is increased be- \\ yond a value $E_{c}$ where the frequency of the soft ripplon vanishes, a phase transition to a \\ spontaneous deformation of the interface in the form of an ordered array of dimples is \\ observed.
}

It has been shown recently that ions can be held at the interface of ${ }^{3} \mathrm{He}-{ }^{4} \mathrm{He}$ mixtures, thus forming a two-dimensional Coulomb system, which in several respects should be similar to the wellstudied layer of electrons on the free surface of liquid He. ${ }^{1,2}$ In particular, some effects which have been predicted as a result of the interaction between the electrons and the free surface s-5 $^{3-5}$ ought to be observable also with ions at the interface, and should even be more pronounced there because the interfacial tension of phase-separated ${ }^{3} \mathrm{He}-{ }^{4} \mathrm{He}$ mixtures is significantly smaller than the surface tension of the liquid-vapor interface of helium.

We have investigated the influence of such a layer of ions on the dispersion relation of interfacial waves (ripplons). It is expected that ripplons with a wave vector around $q=1 / a$ (where $a$ is the capillary length) are considerably lowered in frequency as the electric field $E$ pushing the ions against the interface is increased. ${ }^{4}$ We have experimentally verified this predicted softening in the ripplon dispersion, and moreover have observed an instability connected with it where the macroscopic depression of the interface due to the pressure of the ions breaks up into a regular array of dimples with a "lattice constant" $2 \pi a^{6}$

Without the presence of charges, the dispersion relation of ripplons at the ${ }^{3} \mathrm{He}-{ }^{4} \mathrm{He}$ interface has been found to be well described $b^{7,8}$

$$
\left(\rho_{3}+\rho_{4}\right) \omega^{2}=\left(\rho_{4}+\rho_{3}\right) g q+\sigma_{i} q^{3},
$$

where the terms on the right-hand side are due to gravity and interfacial tension $\sigma_{i}$, respectively. Here $\rho_{4}$ and $\rho_{3}$ are the densities of the ${ }^{4} \mathrm{He}-$ and ${ }^{3} \mathrm{He}-\mathrm{rich}$ phases and $g$ is the acceleration of gravity. On the addition of ions Eq. (1) will be modified, because any local deformation of the interface due to ripplons results in a rearrangement of the originally homogeneous ion distribution, and therefore in an inhomogeneous (wave-vectordependent) ion pressure. This in effect lowers the restoring forces in (1) and hence also the ripplon frequency and leads to an additional term in the dispersion relation ${ }^{4}$ :

$$
\left(\rho_{3}+\rho_{4}\right) \omega^{2}=\left(\rho_{4}-\rho_{3}\right) g q+\sigma_{i} q^{3}-E^{2} q^{2} / 4 \pi 。
$$

Here we have assumed that the interface is completely charged, i.e., the ion density is $n=E / 4 \pi e_{\text {。 }}$ Equation (2) implies a softening of ripplons most obvious for the wave vector $q_{c}=1 / a=\left[\left(\rho_{4}-\rho_{3}\right) g /\right.$ $\left.\sigma_{i}\right]^{1 / 2}$, since in this case $\omega \rightarrow 0$ for $E \rightarrow E_{c}=\left[64 \pi^{2}\left(\rho_{4}\right.\right.$ $\left.\left.-\rho_{3}\right) g \sigma_{i}\right]^{1 / 4}$. For $E>E_{c}, \omega$ becomes imaginary, suggesting that the interface becomes unstable against deformations with wave vector $q_{c}$.

To determine the ripplon dispersion we used a setup schematically shown in the inset of Fig. 1. Located between two capacitor plates, the interface was charged from the top with negative ions (electron bubbles) emitted from a field-emission tip. Since the trapping time of bubbles at the interface becomes very long for temperatures below $T \simeq 0.77 \mathrm{~K}, 2$ a sheet of these bubbles is formed, leading to an indentation of the interface [see Fig. 2(a)] much like the one observed at the free surface by Williams and Crandall. ${ }^{10}$ The interfacial waves were excited by means of a thin horizontal wire mounted slightly below the interface and charged to such a voltage that the ion distribution was not disturbed. ${ }^{11}$ When this voltage was slightly modulated (typically $1 V_{p p}$ ) at a frequency $f$, the interface close to the wire was pulled down periodically due to the ions and interfacial waves with frequency $f$ were generated. The wavelength of these ripplons was determined by scanning the waves with a narrow optical beam as described earlier. ${ }^{7}$

Ripplon dispersion curves obtained in this way are shown in Fig. 1 for three different electric fields at a temperature of $0.567 \mathrm{~K}$. At the lowest field the data points nearly coincide with the dispersion curve for the uncharged interface. As the electric field is increased, softening of the ripplons becomes obvious. At high fields the rip- 


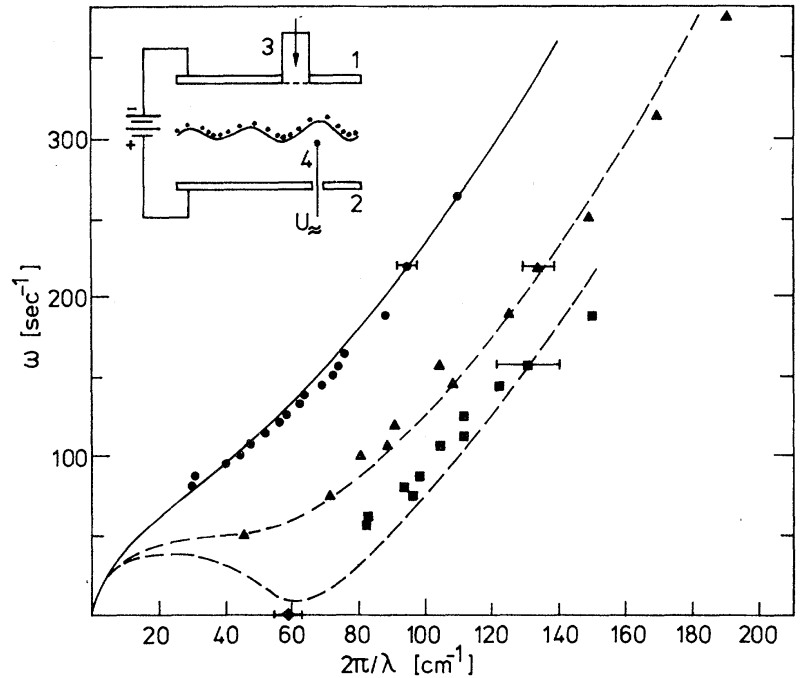

FIG. 1. Dispersion relation of ripplons at the interface of a phase-separated ${ }^{3} \mathrm{He}-{ }^{4} \mathrm{He}$ mixture, completely charged with negative ions from above, at $T=0.567 \mathrm{~K}$. $\bullet, E=111 \mathrm{~V} / \mathrm{cm} ; \Delta, 1000 \mathrm{~V} / \mathrm{cm} ; \boldsymbol{\nabla}, 1119 \mathrm{~V} / \mathrm{cm} ; \bullet$, $1145 \mathrm{~V} / \mathrm{cm}$ (Ref. 9). The dashed curves are calculated according to Eq. (2). The dispersion for the uncharged interface is given by the solid line (Ref. 7). The inset shows the schematical setup: 1,2, top and bottom capacitor plates; 3 , field emission tip; 4 , wave generator. The wave amplitude is largely exaggerated.

plons were strongly damped below $\omega \simeq 40 \mathrm{sec}^{-1}$, and irregular wave patterns appeared, so that no data could be taken in this region. The datum point at $E=1145 \mathrm{~V} / \mathrm{cm}$ and $\omega=0$ is obtained from the periodicity of the spontaneously forming bandlike structures discussed below.

The agreement between the data and Eq. (2) is remarkable: The dashed curves in Fig. 1 indicating the expected behavior contain no adjustable parameter。 It is not self-evident that Eq. (2) should yield such a good description for ripplons in the presence of ions, because in deriving (2) it is assumed that charges at the interface can move freely and follow deformations of the interface immediately. This condition is easily met for electrons at the free surface because of their high mobility of about $10^{6} \mathrm{~cm}^{2} / \mathrm{V} \cdot \mathrm{sec}^{1}$ Negative ions, however, have a mobility of only $\mu=0.025$ $\mathrm{cm}^{2} / \mathrm{V} \cdot \mathrm{sec}$ in the bulk normal phase, ${ }^{12}$ and for ions moving along the interface and interacting with it $\mu$ might be even more reduced。 Still the mobility apparently is high enough for Eq. (2) to apply.

What happens when the electric field is increased beyond the value $E_{c}$ where the instability of the interface is expected? We observe a spon- (a)

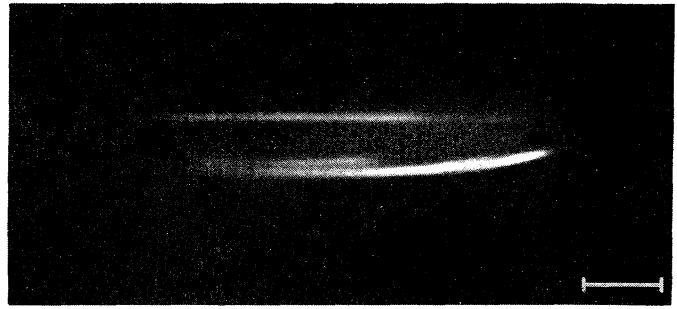

(b)

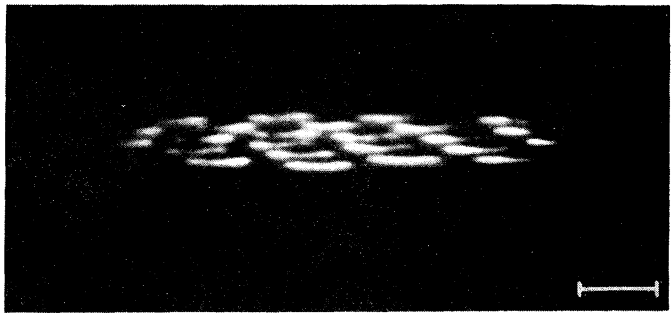

FIG. 2. (a) Deformation of the mixture interface at $T=0.567 \mathrm{~K}$ and $E=1120 \mathrm{~V} / \mathrm{cm}$, observed from below at an angle of $6^{\circ}$. The elliptical bright lines are reflections from the nearly circular rim of the flat indentation. The scale bar represents $1 \mathrm{~mm}$. (b) The electrical field is increased to $E=1160 \mathrm{~V} / \mathrm{cm}$. The large indentation has spontaneously deformed into an array of smaller dimples.

taneous deformation of the interface which within fractions of a second increases until charges break through. ${ }^{13}$ Since lost charges are resupplied by the ion source, the interface is continuosly stirred by this process. After the field emission source is switched off, the breakthrough stops, with still some charge remaining at the now calm interface. The structure of the interface in this case is strikingly different from that at $E<E_{c}$ : The flat homogeneous indentation $(\simeq 1$ $\mathrm{cm}$ in diameter) at lower fields has broken up into a lattice of dimples with a distance of about 1 mm [see Fig. 2(b)].

This phase transition from a homogeneous to a structured indentation can also be observed without a breakthrough occurring when the interface is charged at $E<E_{c}$ and then the field is slowly raised at constant charge density (i.e., with the ion source switched off). At a field $E_{c}{ }^{\prime}$ (slightly higher than $E_{c}$ ) first a corrugated bandlike structure appears, apparently closely related to the soft ripplon - and exactly with the same wavelength -and these bands already during formation break up further into individual dimples, which again form the static regular array of Fig. 2(b). The symmetry of this "dimple crystal," although somewhat difficult to determine because of the very oblique viewing angle, appears to be 
hexagonal. ${ }^{6}$ Directions of the crystal axes were arbitrary and slowly drifting with time, which excludes a relevant influence of the rectangular chamber walls on crystal formation. Frequently imperfections like step dislocations were observed. When the electric field was decreased again below $E_{c}{ }^{\prime}$, the dimple crystal "melted" without noticeable hysteresis.

We finally note that the dimples making up the lattice are found to exist also as individual isolated entities if the charge density is low. Since each dimple holds about $10^{6}$ negative ions, these dimples strongly repel each other as a result of Coulomb interaction. The dimple lattice can therefore be regarded as a kind of macroscopic two-dimensional Wigner crystal. ${ }^{14-16}$ Interestingly enough, the ion density in each dimple is so high that the individual ions there might also form a two-dimensional crystal, however with a lattice constant three orders of magnitude smaller. It is a pleasure to thank H. Kinder for valuable discussions, and $\mathrm{W}$. Bosch and T. Rapp for experimental assistance. This work was supported by the Deutsche Forschungsgemeinschaft.

\footnotetext{
${ }^{1}$ For a review see, e.g., C. C. Grimes, Surface Sci. $\underline{73}, 379$ (1978).

${ }^{2}$ P. Leiderer, M. Wanner, and W. Schoepe, in Proceedings of the Fifteenth International Conference on Low Temperature Physics, Grenoble, France 23-29
}

August 1978 (to be published).

${ }^{3}$ V. B. Shikin, Zh. Eksp. Teor. Fiz. 58, 1748 (1970)

[Sov. Phys. JETP 31, 936 (1970)].

${ }^{4}$ L. P. Gorkov and D. M. Chernikova, Pis'ma Zh.

Eksp. Teor. Fiz. 18, 119 (1973) [JETP Lett. 18, 68

(1973)].

${ }^{5}$ K. Mima, H. Ikezi, and A. Hasegawa, Phys. Rev. B 14, 3953 (1976).

${ }^{6}$ L. P. Gorkov and D. M. Chernikova, Dokl. Akad. Nauk SSSR 228, 829 (1976) [Sov. Phys. Dokl. $\underline{\text { 21 }}, 328$ (1976)].

${ }^{7}$ P. Leiderer, H. Poisel, and M. Wanner, J. Low

Temp. Phys. 28, 167 (1977).

${ }^{8}$ In a more accurate treatment damping of the ripplons has to be taken into account (see, e.g., Ref. 7).

${ }^{9}$ The fields were not measured directly, but calculated from $E=U / d$, where $U$ is the voltage between the capacitor plastes and $d=2.7 \mathrm{~mm}$ is the distance of the interface from the bottom plate.

${ }^{10}$ R. Williams and R. S. Crandall, Phys. Lett. 36A, 35 (1971).

${ }^{11}$ This was easily achieved by adjusting the wire potential such that the interface was not distorted in the vicinity of the wire.

${ }^{12} \mathrm{P}$. Leiderer and M. Wanner, Phys. Rev. Lett. 41 , 480 (1978).

${ }^{13} \mathrm{~A}$ similar instability has been observed with electrons at the free surface of liquid helium by M. S. Khaikin, in Proceedings of the Fifteenth International Conference on Low Temperature Physics, Grenoble, France 23-29 August 1978 (to be published).

${ }^{14}$ R. S. Crandall and R. Williams, Phys. Lett. $\underline{34 \mathrm{~A}}, 404$ (1971).

${ }^{15}$ P. M. Platzman and H. Fukuyama, Phys. Rev. B $\underline{10}$, 3150 (1974).

${ }^{16}$ R. W. Hockney and T. R. Brown, J. Phys. C $\underline{8}, 1813$ (1975). 
(a)

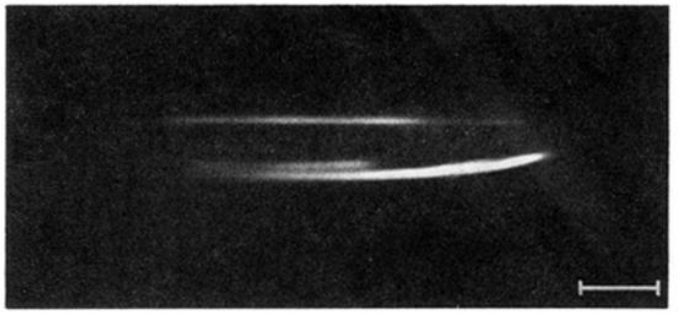

(b)

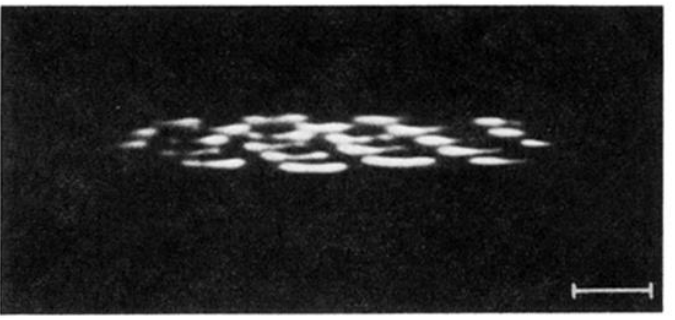

FIG. 2. (a) Deformation of the mixture interface at $T=0.567 \mathrm{~K}$ and $E=1120 \mathrm{~V} / \mathrm{cm}$, observed from below at an angle of $6^{\circ}$. The elliptical bright lines are reflections from the nearly circular rim of the flat indentation. The scale bar represents $1 \mathrm{~mm}$. (b) The electrical field is increased to $E=1160 \mathrm{~V} / \mathrm{cm}$. The large indentation has spontaneously deformed into an array of smaller dimples. 